\title{
ESTAR + FC en el castellano peruano
}

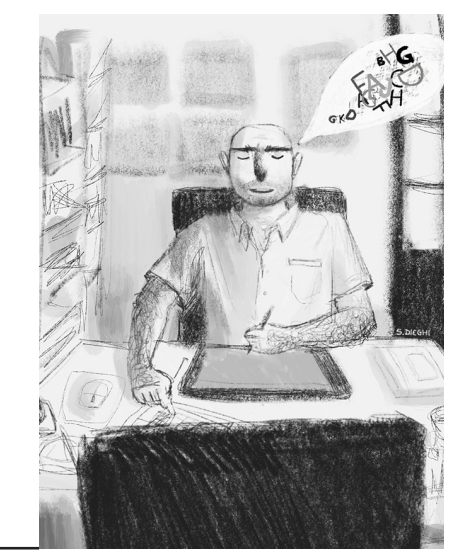

Víctor Arturo Martel Paredes

Departamento de Lingüística de la Facultad de Letras y Ciencias Humanas,

Universidad Nacional Mayor de San Marcos, Perú

vmartelp@unmsm.edu.pe

Trabajo recibido el 24 de marzo de 2019 y aprobado el 16 de junio de 2019.

\section{Resumen}

En el presente artículo, describiré la construcción de tipo Ella está que canta del castellano de la variedad peruana, en donde el verbo 'estar' selecciona una Frase Complementante (en adelante FC) para conformar una predicación compleja. Seguiré la propuesta de Cuervo (2003) sobre la conformación de estructura eventiva para analizar los componentes de dicha construcción; y la ausencia de rasgos de chequeo de Caso en FT para licenciar el hiperascenso de Frase Determinante (FD) (Martins y Nunes 2010; Fernandez-Salgueiro 2005; Fong 2019, Pesetsky y Torrego 2001, 2004, 2007). Probaré, en primer lugar, la monoclausalidad de la estructura. Luego, mostraré que esta estructura se conforma a través de una organización eventiva por complementación: un evento estativo selecciona a otro dinámico, y ambos son interpretados desde una perspectiva aspectual de iteración; y, finalmente, explicaré el ascenso del sujeto desde una cláusula inferior a través de la inespecificación de rasgo $\mathrm{T}$ del verbo inferior, con el consecuente "escape" de la FD a un nivel superior, y, para ello, asumiré la posibilidad de considerar Esp,C como posición argumental o mixta A/A', dadas las propiedades de selección de ESTAR.

\section{ESTAR+CP in Peruvian Spanish}

\begin{abstract}
In this paper, I will describe the construction Ella está que canta ('She is that (she) sings') from the Peruvian variety of Spanish, where 'estar' selects a CP to conform a complex predication. I will follow Cuervo's (2003) eventive structure in order to analyse the components in this construction; and the absence or underspecification of $\mathrm{T}$ in the embedded clause which allow the raising of DP through CP to Spec,T from the matrix clause (Martins \& Nunes 2010; Fernandez-Salgueiro 2005; Fong 2019, Pesetsky \& Torrego $2001,2004,2007)$. I will prove, in the first place, the monoclausality of the
\end{abstract}

\section{Palabras clave \\ Hiperascenso Concordancia predicación compleja castellano peruano}

Keywords

hyperraising agreement complex predication Peruvian Spanish 
structure. Then, I will show that this structure is made through an organization of events by complementation: a state event selects a dynamic one, and both are interpreted from an aspectual perspective of iteration; finally, I will explain the rising of the subject from a lower clause through the feature nonspecification of $\mathrm{T}$ of the lower verb, with the consequent "escape" of the DP to a higher level, and, for this, we assume the possibility of considering Esp, C as argument position or mixed A / A ', given the selection properties of ESTAR.

\section{ESTAR + FC do castelhano da variedade peruana}

\section{Resumo}

No presente artigo, vou descrever a construção do tipo Ella está que canta do castelhano da variedade peruana, onde o verbo 'estar' seleciona uma FC para formar uma pregação complexa. Seguirei a proposta de Cuervo (2003) sobre a conformação de eventual estrutura para analisar os componentes da referida construção; e a ausência de recursos de verificação de caso no FT para licenciar a hyperase de Determining Phrase (FD) (Martins \& Nunes 2010; Fernandez-Salgueiro 2005; Fong 2019, Pesetsky \& Torrego 2001, 2004, 2007). Provaremos, em primeiro lugar, a monoclausalidade da estrutura. Então, veremos que essa estrutura é formada através de uma eventual organização por complementação: um evento estacionário seleciona um evento dinâmico e ambos são interpretados a partir de uma perspectiva aspectual de iteração; e, finalmente, a ascensão do sujeito a partir de uma cláusula inferior será explicada através da não especificação do traço T do verbo inferior, com a consequente "fuga" do DF para um nível superior, e, para isso, assumimos a possibilidade de considerar Esp, C como posição de argumento ou A / A misto, dadas as propriedades de seleção de ESTAR.

\section{Introducción}

En este artículo me ocuparé en describir y analizar la construcción del español peruano "estar+FC". En primer lugar, mostraré cuáles son las propiedades de la construcción. En esta primera parte presentaré de qué manera se conforman estructuras gramaticales y qué formas la transgreden. Luego, en la segunda parte, presentaré pruebas de monoclausalidad; es decir, su lectura aspectual depende de la complementación de dos predicados. En la tercera parte, revisaré algunos conceptos teóricos que guíen al análisis de esta construcción, como la definición de predicado complejo, restructuración y el "estar" predicativo. A partir de ello, realizaré el análisis, basándome también sobre propuestas teóricas sobre valuación de rasgos interpretables. Finalmente, brindaré conclusiones sobre el estudio.

\section{Propiedades de la construcción ESTAR+FC}

La construcción que será objeto de análisis está conformada por un verbo principal ESTAR, verbo auxiliar de contenido aspectual no perfectivo, el cual, si bien es cierto recibe la flexión de Tiempo, más adelante mostraré que existen restricciones con respecto a los Tiempos y Modos con los que se puede construir este enunciado. Por otro lado, ESTAR selecciona un complemento, encabezado por la partícula subordinante 'que'. El complemento
Palavras-chave:

yperascenso Concordância pregação complexa castelhano peruano 
encabezado por 'que' cosiste en una forma oracional plena, es decir, con flexión de Tiempo y, en consecuencia, con un Sujeto. No obstante, como explicaré, el sujeto no puede ocurrir fonéticamente en este dominio. A continuación, presento las características de esta construcción con mayor detalle.

\subsection{Sujeto}

El sujeto incrustado tiene que ser nulo (1c) y su antecedente, un sujeto independiente sintácticamente en la oración matriz expresado fonéticamente, como (1a), o no, como (1b).

(1) a. Luisa, está que pro, canta.

b. pro $_{i}$ está que pro ${ }_{i}$ canta.

c. Luisa está que (*ella/*Luisa) canta.

En (1c) ocurre la agramaticalidad por la aparición del sujeto expresado fonéticamente en la oración subordinada, tanto duplicado como expresado en forma pronominal ${ }^{1}$. Aunque compartan el mismo sujeto, una construcción donde pro c-comanda a la FD ligada a él sería agramatical:

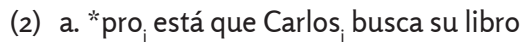

b. Carlos está que busca su libro.

Por otro lado, no haber una selección temática de sujeto agente en este tipo de construcciones; es decir, ESTAR refleja las propiedades temáticas del verbo incrustado:

(3) a. Meteorológico

Está que llueve.

b. Haber impersonal

*Está que hay zancudos.

c. No agentivos

Está que me duele la cabeza.

La única agramaticalidad observada es (3b) por contravenir la aspectualidad. La aspectualidad es la forma en la que se desarrolla el evento descrito por la frase verbal de la construcción. De ello hablaré más adelante.

\subsection{El tiempo}

Los rasgos de tiempo deben ser compartidos entre el verbo de la oración principal y el de la oración incrustada. En 4 presento ejemplos gramaticales donde ambas cláusulas comparten Tiempo Presente del Modo Indicativo (4a), del Tiempo Pasado Imperfecto (4b); y agramaticales, donde las cláusulas muestran tiempos distintos (4c y $4 \mathrm{~d}$ ).

(4) a. María está (PRESENTE) que camina (PRESENTE) a su casa.

b. Camilo estaba (P. IMPERFECTO) que tomaba (P. IMPERFECTO) mucha cerveza.

c. *María está (PRESENTE) que caminará (FUTURO) a su casa.

d. *Camilo estaba (P. IMPERFECTO) que tomó (P. PERFECTO) mucha cerveza.

Sobre el tiempo expresado en estas construcciones, es notable la restricción del tipo de información temporal para la construcción de oraciones gramaticales con la estructura ESTAR+FC, pues, como indiqué en la definición
1. Sin embargo, las siguientes oraciones, donde el núcleo FT se ha movido a posición de Foco (a) y otra en la que se realiza de manera forzada el pronombre (b), son posibles:

a. Está Luisa que come hamburguesas. b. ??Luisa está (ELLA) que canta. No obstante, estas ocurrencias no son cruciales para el análisis. 
líneas arriba sobre la aspectualidad no perfectiva de ESTAR, la especificación de pretérito perfecto (5a), pluscuamperfecto (5b) o futuro perfecto (5c) están descartados como gramaticales.

(5) a. "María ha estado que ha buscado su libro.

b. *Esteban había estado que había llorado.

c. "Luis habrá estado que habrá tocado la puerta.

Por otro lado, el modo indicativo es el usual en estas construcciones, aunque el subjuntivo presente también es aceptable:

(6) Dudo que ella esté que estudie

Una posible respuesta, aunque tentativa, es que las formas compuestas de (5) y (7a), y de modos condicional (7b) o imperativo (7c) son agramaticales debido a que se oponen no solo a la aspectualiad interna de ESTAR, como en el caso de 5a, sino a la estructura epistémica de enunciado: el conocimiento del hablante sobre el evento es un hecho logrado, y no una posibilidad ni potencialidad. Por tanto, formas condicionales, futuras o imperativas debilitan o se contraponen a estructuras de las que se tiene dominio, por tanto, son agramaticales.

(7) a. *Ha estado que te ha buscado. (Pasado compuesto)

b. "Miguel estaría que te llamaría. (Modo condicional)

c. "Estate que estudies. (Modo imperativo)

\subsection{Resumen}

Resumo a continuación lo visto hasta ahora:

(i) La construcción presenta el mismo sujeto tanto para el verbo ESTAR como para el verbo de la oración incrustad (incluso presenta las mismas propiedades temáticas del verbo incrustado).

(ii) El tiempo es el mismo tanto en el verbo ESTAR como en el verbo incrustado.

(iii) La construcción admite tiempo presente y pretérito imperfecto.

(iv) La construcción es agramatical cuando el tiempo es compuesto y el modo no es indicativo.

\section{Pruebas de constitución}

En esta sección brindaré pruebas de que la estructura que analizo es un predicado complejo. Para ello, en primer lugar, mencionaré cómo recogí los juicios de gramaticalidad/agramaticalidad. Luego, detallaré los ejemplos que me servirán para la prueba de monoclausalidad.

\subsection{Metodología de trabajo}

Con el objetivo de dar cuenta de la constitución de la estructura ESTAR+FC, sometí a juicio de gramaticalidad a distintos hablantes de castellano peruano. Los requisitos que debían reunir eran (a) ser nativo hablante, (b) habitar en Perú (específicamente en la región Lima), (c) tener más de 15 años de 
edad y (d) ser conocedor de la estructura bajo estudio y emplearla frecuentemente. De esta manera, bajo estas consideraciones, hice una encuesta a diez participantes para conocer sus juicios gramaticales sobre ciertas transformaciones y determinar: (1) dependencia de tiempo y sujeto entre el verbo principal y el "subordinado", y otros tiempos posibles, y (2) la constitución de ESTAR+FC.

\subsection{Monoclausalidad}

A diferencia de FC ensambladas con verbos predicativos, como decir, pensar, sostener, etc., la construcción ESTAR+FC es una estructura monoclausal con un único sujeto obligatorio y una sola especificación de Tiempo en ambos verbos. A continuación, contrastaré verbos de complemento proposicional (p. e., ver, oír, etc.) con la construcción ESTAR+FC con el fin de señalar que, mientras es posible comprobar la independencia de FC para los primeros, esto no lo es para ESTAR como verbo matriz.

\section{a. Dislocación del foco}

En (8b), cuando la FC se mueve a la izquierda de la oración por Foco, surge una interpretación forzada, sin la lectura aspectual de la frase original; por tanto, (8b) es agramatical en el sentido de que no se puede recuperar la aspectualidad del enunciado original. Compárese con (8a) donde no hay contenido aspectual por ser una construcción propositiva; por tanto, luego del movimiento, la interpretación sigue siendo gramatical.

(8) a. [que Juan bailaba] decía María. (C. Proposicional)

b. *[Que canta] está Ramiro. (ESTAR+que)

\section{b. Clítico neutro $10^{2}$}

En (9b), una separación de ESTAR de FC resulta en ESTAR con un significado de existencia de la cosa referida por la FD, no la información aspectual del evento que está en transcurso cuando se adjunta a la FC.

(9) a. He dicho [que María bailaba con Juan]. Pedro también lo dijo. (C. Proposicional) b. María está [que camina]. Pedro también lo está. (ESTAR+que)

\section{c. Respuesta}

De igual manera, en la respuesta de FC se pierde la lectura aspectual de la construcción.

(10) a. -¿Qué dijiste ayer por la noche?

- Que [Juan corría por el parque]. (C. Proposicional)

b. -¿Qué estaba Nicolás? /¿Cómo estaba Nicolás?3

-*Que jugaba. (ESTAR+que)

\section{d. Cláusula escindida}

La construcción al escindirse resulta en anómala a diferencia de una construcción con verbo propositivo.

(11) a. [Que Juan corría] fue lo único que dije. (C. Proposicional) b. ??[Que marchaba] es lo único que Carlos estaba. (ESTAR+que) 2. o neutro es adecuado solo para
predicados de contenido nocional (Fernández Leborans 1995, 254)

3. La pregunta con "cómo" sería más adecuada en el sentido de que el predicativo que sigue a ESTAR usualmente es asumido como un adverbial. 


\section{e. Cláusula pseudoescindida}

Esta vez, la construcción resulta peor que en el anterior caso.

(12) a. Lo único que dije fue [que Juan corría] (C. Proposicional)

b. "Lo único que María está es [que corre]. (ESTAR+que)

\section{f. Coordinación}

La primera FC con ESTAR denota las aspectualidad que ayuda a interpretar la segunda FC en el mismo sentido.

(13) a. He dicho [que Juan corría] y [que María saltaba]/He dicho que Juan corría y María saltaba. (C. Proposicional)

b. [Juan está que corre] y [que suda]. (ESTAR+que)

\section{g. Construcción no solo...sino también}

La construcción es también vista como gramatical, pero los hablantes indican que sería mejor mencionar ESTAR otra vez antes del segundo FC en vez de omitirlo

(14) a. No solo dije [que Juan bailaba] sino también [que bebía]. (C. Proposicional)

b. ??No solo Marco está [que baila] sino también [que ríe]. (ESTAR+que)

Después de estas observaciones, puedo concluir que, además de las dependencias de tiempo, ESTAR+FC es una sola construcción compleja, la cual no puede ser dividida en dos constituyentes, en oposición al propositivo con verbos principales agentivos, porque ESTAR funciona como auxiliar que ayuda al FC a ser interpretado como evento en progreso, entonces ESTAR porta rasgos aspectuales que deben ser adjuntados al predicativo FC.

Adicionalmente a las pruebas mostradas, la estructura se comporta como un complejo predicativo entero a través de la modificación adverbial:

\section{h. Modificación adverbial}

Los adverbios modifican a toda la estructura ESTAR+FC y no solo a ESTAR $\mathrm{o}$ al verbo incrustado independientemente. Esto se observa cuando el adverbio expresa una modificación al desarrollo eventivo del verbo incrustado (15b):

(15) a. Varias veces/frecuentemente/a cada rato estoy que me duermo. (modifica a ESTAR+FC)

b. *Una sola vez/de vez en cuando/está que lee. (modifica a 'leer')

c. "Quietamente/ más o menos/casi estoy que me duermo. (modifica a 'estar')

En 15a la frase adverbial afecta al evento descrito por el verbo incrustado siempre y cuando coincidan en la aspectualidad; precisamente, $15 \mathrm{~b}$ no coincide con la estructura aspectual del verbo incrustado por lo que la estructura es agramatical.

Por último, las formas negativas afectan a toda la estructura y no solo al verbo principal (16a y 16b). La aparición de la negación en la oración incrustada, ante el verbo, deviene en agramaticalidad (16c). 
(16) a. No estaba que lloraba.

b. ?Nunca está que estudia.

c. *Estabas que no bailabas.

i. Frases idiomáticas

Aunque para el caso bajo estudio puedo asegurar de que se trata de un ascenso de FD incrustado a Esp, T de la oración matriz, existe una prueba sobre lecturas de frases idiomáticas para determinar si la FD en cuestión sube a una posición A (hyper raising) o se posiciona en A' por topicalización ${ }^{4}$. De darse el segundo caso, la lectura idiomática se perdería. Por ejemplo:

Portugués (Martins y Nunes 2010)

(17) a. A vaca foi pro brejo.
La vaca fue para la ciénaga
Lectura idiomática: las cosas van mal
Lectura literal: la vaca fue a la ciénaga

b. A vaca, o João disse que foi pro brejo. La vaca João dice que fue para la ciénaga

*Lectura no idiomática: João dice que las cosas van mal Lectura literal ('Juan dice que la vaca fue a la ciénaga.'): OK

c. A vaca parece que foi pro brejo.

La vaca parece que fue para la ciénaga

Lectura idiomática ('parece que las cosas van mal.'): OK

Lectura literal 'parece que la vaca fue a la ciénaga'

Español (Fernández Sánchez 2015, 6)

(18) a. [Mala hierba]i nunca muere ti.

b. [Mala hierba]i, mi padre dice que nunca muere ti. (lectura no idiomática)

c. Mala hierba parece que nunca muere. (lectura idiomática OK)

Ahora, presento ejemplos con la estructura para examinar si se mantiene la lectura idiomática:

(19) a. La curiosidad mató al gato.

b. La curiosidad está que mata al gato. (lectura idiomática OK)

c. La curiosidad, mi padre dice que mató al gato. (lectura idiomática ¿??)

Por tanto, la interpretación idiomática en (19b) se mantiene, la cual involucra un movimiento a posición A; por otro lado, en el movimiento a una posición $A^{\prime}$ de (19c) la interpretación se desvía aunque no drásticamente.

\subsection{Resumen}

(i) La estructura ESTAR+FC tiene una lectura aspectual de reiteración.

(ii) En las pruebas de monoclausalidad, en 9b 'lo neutro' es posible obtener una lectura gramatical al dividir el verbo ESTAR de la estructura encabezada por 'que'; no obstante, la lectura aspectual se pierde, tomando la lectura predicativa tradicional de 'logro alcanzado' (Fernández 1995). De manera adicional, la modificación adverbial afecta a la estructura ESTAR+FC y no solo al verbo matriz.
4. No obstante, Fernández Sánchez (2015) critica esta prueba aduciendo que las construcciones en las que hay un FD en posición A' también pueden conservar su sentido tradicional. Solo añadiré esta prueba al conjunto ya presentado para complementar la idea de monoclausalidad de la estructura. 
(iii) ESTAR +FC funcionan como un solo predicado complejo.

Una vez comprobado que se trata de estructura compleja; analizaré de qué manera los dos núcleos se constituyen en una sola frase.

\section{Predicados complejos y su conformación}

Para analizar la estructura que observamos, primero veremos algunos conceptos que han sido desarrollados sobre la predicación y sobre el verbo ESTAR específicamente.

\subsection{Predicación compleja}

Los predicados complejos son definidos como estructura verbales que contienen al menos dos morfemas, cada uno marcando un argumento en la rejilla temática (Baker 1996, citado en Lomashvili 2011, 1) aunque Baker limita las construcciones a causativos, aplicativos y ascenso de posesor. Siguiendo a Bril (2004, 2-3), los predicados complejos (o también llamado seriación de verbos) cumplen con ciertas características a través de las lenguas:

a) Los verbos y frases verbales (o predicados o núcleos) constituyen una sola predicación que refieren a aspectos de un solo evento;

b) Estos núcleos complejos conforman una oración, sin límite oracional o marcador de dependencia;

c) Constituyen una sola entidad prosódica, sin pausa;

d) Comparten al menos un argumento sintáctico o un solo conjunto de argumentos;

e) Comparten el mismo tiempo, aspecto, modo, (marcados como $V_{1} \circ V_{2}$ ) la misma fuerza ilocucional, la misma polaridad afirmativa o negativa, y el alcance de la negación cubre todos los núcleos;

f) No hay pérdida de acento o forma fonológica; no se pierde las propiedades morfosintácticas o semánticas de los núcleos;

g) La autonomía léxica es un prerrequisito para la seriación; de lo que se excluyen los co-verbos no autónomos y formas no finitas, así como compuestos co-lexicalizados.

Sobre el punto (f), el verbo ESTAR, si bien es cierto que aparece independientemente en otras estructuras, suele expresarse en formas más abreviadas, como 'ta que te busca' por 'está que te busca' o 'taba que lloraba' por 'estaba que lloraba'. Sobre los demás puntos concuerdo plenamente. En resumen, la construcción es un predicado complejo que constituye una sola unidad gramatical.

\subsection{Conformación de Estar predicativo y FC: ¿unidad compleja por restructuración?}

Fernández Leborans (1995), en cuanto a la estructura de las oraciones con ESTAR, sostiene que la definición aspectual que alcanza ESTAR se logra léxicamente, antes de la derivación sintáctica, junto al complemento predicativo. Es así que el predicativo no complementa como argumento al verbo, sino que, en calidad de predicativo, lo califica y especifica aspectualmente. Así, Fernández propone que antes de la sintaxis habría un proceso fusión de los eventos que expresan ESTAR y del predicativo. El predicativo se legitimaría mediante la fusión entre su propia posición eventiva $<$ e $>$ con la 
posición eventiva <e> del predicado matriz: en Juan está triste no es simple conexión entre posición $<\mathrm{e}>$ de ESTAR y $<\mathrm{e}>$ de triste, sino unificación de las dos posiciones en una sola. Finalmente, el sujeto, luego del proceso de fusión de dos eventos en uno, es adecuadamente interpretado como sujeto temático de un verbo complejo.

De acuerdo con Wurmbrand (p.c.) su estudio tipológico demuestra que la restructuración por medio de la incorporación o “fusión” en las lenguas es posible a través de dominios $\mathrm{V}$ y v, pero que se mantenga una transparencia de dominios a través de una FC no tiene sustento. En ese sentido, la autora (Wurmbrand 2015, 2018) señala que hay una jerarquía hipotética universal implicacional de restructuración en que los complementos de actitud/habla son tipos oracionales sintácticamente más complejos, más independientes y menos transparentes; los complementos de futuro, menos complejos, menos independientes y más transparentes; y los complementos atemporales, los menos complejos y más transparentes. Una prueba de transparencia de dominios y que muestra la restructuración de núcleos verbales en una sola unidad predicativa es el ascenso de clíticos. Basta con observar que no es posible el ascenso de clíticos en la estructura que nos ocupa:

(20)a. María quiere buscarlo. b. María lo quiere buscar.

(21) a. María está que lo busca. b. *María lo está que busca.

Aunque los casos típicos de restructuración son los infinitivos para el español y otras lenguas, donde es posible el ascenso de clíticos a través de predicados léxicos (verbos) (20a-b), los ejemplos de (21a-b) muestran que el paso de clíticos de un dominio FC a otro no es posible; es decir, la FC bloquea la restructuración (Wurmbrand 2018) ${ }^{5}$.

\subsection{Conformación por complementación: vBE+vDO (Cuervo 2003)}

Otro alcance para explicar la predicación compleja es la complementación. Cuervo (2003) sostiene su argumentación sobre la estructura eventiva como principio organizativo, el cual provee una explicación del licenciamiento de sintáctico y semántico de argumentos: si los argumentos son licenciados como participantes de eventos, entonces su licenciamiento depende crucialmente del tipo de evento expresado por el verbo. Para la autora, los argumentos son licenciados sobre la base de las estructuras eventivas que son posibles dadas las relaciones de complemento y especificador, y de tres tipos de núcleos: introductores de eventos (v ligera), introductores de argumentos (Voz y Aplicativo) y las Raíces.

Siguiendo a Marantz (1997), los verbos son formados en la sintaxis a través de la combinación de una raíz léxica y un núcleo verbalizador v ligera. Asimismo, la autora sigue a Harley (1995) en la idea de que hay tres tipos de $\mathrm{v}$ ligera que corresponden uno a uno a tres tipos básicos de eventos, es decir, las raíces y las v ligeras se combinan sintácticamente para construir predicados eventivos. Estos son los siguientes:
5. Sin embargo, el complementante "que" no invalida el ascenso de clíticos: Tengo que hacerlo. o tengo que hacer.

Como afirma Bosque y Gutiérrez (2008: 608), la restricción previene el ascenso de clíticos desde el "interior de una oración flexionada", es decir, con especificación de Tiempo y rasgos $\varphi$. 


\begin{tabular}{|c|c|c|}
\hline Tipos de $v$ & Tipos de eventos simples & Ejemplos \\
\hline vDO & Actividades & Bailar, barrer, correr \\
\hline vGO & Cambios & Caer, ir, morir, crecer \\
\hline vBE & Estados & Gustar, admirar, faltar \\
\hline
\end{tabular}

Tabla 1. Descripción de núcleos v según el evento

Siguiendo con la exposición, Cuervo asume que los eventos complejos pueden ser formados a través de la combinación de dos eventos predicativos.

\begin{tabular}{|c|c|c|}
\hline Posibles combinaciones & Tipos de eventos complejos & Ejemplos \\
\hline vDO+vDO & Causativos & Hacer lavar, hacer reñir \\
\hline vDO+vGO & Causativos & Hacer crecer, hacer caer \\
\hline vDO+vBE & Causativos & Romper, quemar, cerrar \\
\hline vGO+vBE & Incoativos & Romperse, quemarse cerrarse \\
\hline
\end{tabular}

Tabla 2. Combinación de núcleos eventivos

Cuervo sostiene además que su aproximación es capaz de configurar cualquier estructura causativa o incoativa (vDO o vGO) que incruste una relación de predicación que sea consecuencia del evento dinámico más alto. Así, abarca construcciones conformadas por un núcleo verbal funcional y predicados, expresados como verbo y adjetivos; verbo y preposición, verbo y partículas o verbos derivados de adjetivos.

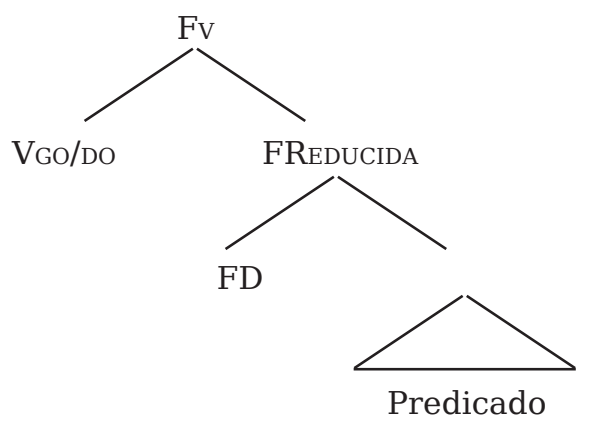

Fig. 1. Conformación de predicado complejo vDO/GO + Cláusula Reducida

Si deseara aplicar la propuesta de Cuervo a la estructura, se presentaría la dificultad de establecer una correspondencia de "resultado" con la FC, es decir, el evento dinámico que es originado por el predicado estativo (vBE) no se corresponde con la naturaleza resultativa de la propuesta: la FC describe un evento dinámico por sí mismo, el cual puede estar constituido por subeventos, los que producen una interpretación de evento causativo o incoativo, y este, a su vez, deben ensamblarse con una predicación superior con su propia estructura eventiva.

Una respuesta a ello es que Cuervo no tuvo en consideración en su tipología la combinación de subeventos vBE+vDO; es decir, asumió que el resultado de un estado debe ser siempre un atributo o estado carente de dinamicidad. Propongo, entonces, que (i) la estructura no necesariamente debe configurarse en una predicación superior cuyo complemento es su resultado; sino que la interpretación de ambos eventos es la suma de una predicación estativa más una predicación dinámica, lo que se interpreta en una 
prolongación de la realización del evento inferior; y (ii) el evento que despliega la complementación predicativa es de actividad (vDO) simple y no compuesta (incoativa, causativa), ya que la lectura interpretativa que se haga de esta en combinación con la predicación matriz ESTAR (vBE) es de ITERACIÓN ${ }^{6}$. Esto es observable en los siguientes casos, donde se hace patente la lectura aspectual iterativa de estar+FC y la imposibilidad de admitir eventos incoativos o causativos (los cuales implican un estado terminado o resultado):

\section{(22) Actividad}

a. Lucía está que come manzanas. (-télico)

b. Lucía estaba que comía una/la manzana. (-télico)

(23) Realización

a. *Lucía estuvo que corrió 100 metros. (+télico)

b. *Lucía ha estado que ha comido una/la manzana/manzanas. (+télico)

(24) Logro

a. *Él está que encuentra su corbata.

b. *Alberto está que llega a la reunión.

Pero

c. La noticia está que llega a todas partes. (-télico/actividad)

(25) Estado

a. "Laura está que parece desesperada.

b. *Laura está que quiere una galleta.

Pero

c. Laura está que quiere irse. (-télico/actividad)

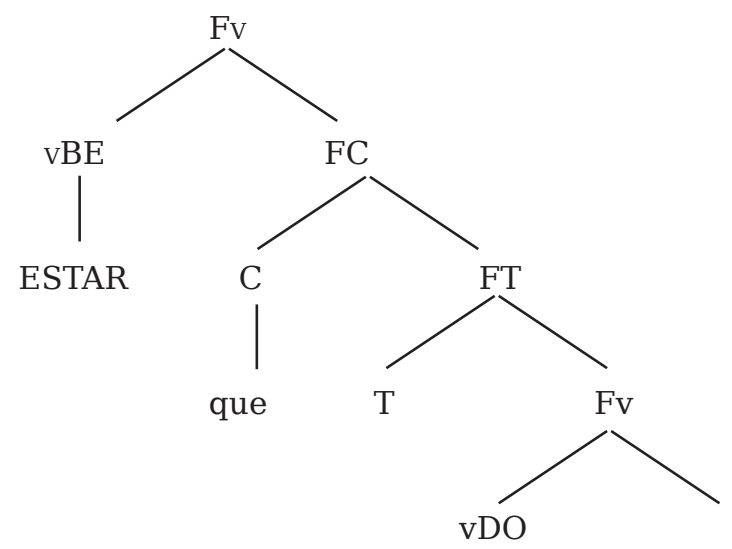

Fig. 2. Propuesta de conformación de predicación compleja sobre la base de Cuervo (2003)

Con esta construcción $\mathrm{vBE}+\mathrm{FC}(\mathrm{vDO})$ la interpretación que se obtiene es de iteración del evento de actividad, pues implica un estado o duración en el que una actividad se desarrolla de manera prolongada o repetida. Para esta propuesta, es necesario explicar de dónde nace el sujeto y sus posteriores movimientos; y cómo ambas predicaciones comparten los mismos rasgos temporales.
6. Cuervo (2003) sugiere que la combinación *vBE + vGO/vDO serían imposibles o agramaticales, ya que los eventos que servirían de complementación al estativo vBE tendrían que ser estados primero (nota de pie de página, 18). 


\section{Análisis}

Concluí del apartado anterior que la predicación compleja bajo estudio no es un caso de restructuración, donde se incorpora un núcleo léxico a otro; propongo, siguiendo a Cuervo (2003), el ensamble de dos tipos verbales con rasgos eventivos propios a través de la complementación, lo cual genera una interpretación aspectual de iteración o duración, como una sola predicación. Para el análisis, trataré de explicar el lugar de generación del sujeto. La propuesta es que este se genera en la predicación incrustada y asciende para recibir caso del verbo ESTAR.

\subsection{Esp,C como posición argumental (o mixta)}

Fong (2019), para el análisis de hiperascenso de FD en la lengua mongol, asume que el Especificador de $\mathrm{C}$ no tiene por qué ser inherentemente una posición $\mathrm{A}^{\prime}$, es decir, podría ser una posición argumental. La autora explica que el sujeto de la oración incrustada tiene caso acusativo en (26b) (en comparación con la posibilidad más esperable de asignación de caso nominativo, como en (26a)), gracias a que este se encuentra en un dominio que es accesible a la computación: Esp de C:

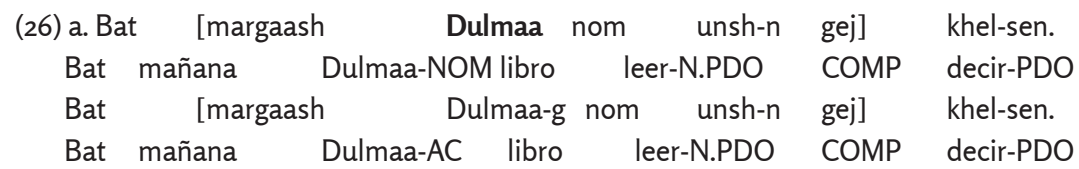

'Bat dijo que Dulmaa leerá un libro mañana'

Por tanto, hay una relación entre la v matriz y el sujeto incrustado, a través de una FC, que constituye una fase. Fong asume que el complementante de una oración finita puede estar dotada de rasgos $\varphi$. Estos activan el movimiento de la meta apropiada más cercana: el sujeto incrustado, al especificador de FC. Desde esa posición, el sujeto incrustado puede recibir caso acusativo de la v matriz sin incurrir en una violación de localidad. Para ello, la autora sostiene crucialmente que la FD se mueve del especificador de FT y luego aterriza en el especificador de FC; es decir, la posición del Especificador de FC sería argumental. Esta misma afirmación la hace Wurmbrand (2018), quien cita a van Urk (2015) en un estudio sobre la lengua dinka, donde observa que el movimiento Especificador de $\mathrm{C}$ tiene propiedades A y A'. De esta manera, los núcleos $\mathrm{C}$ pueden tener rasgos $\mathrm{A}$ además de los usuales rasgos $\mathrm{A}^{\prime}$. Si $\mathrm{C}$ tiene rasgos $\mathrm{A}$, como rasgos $\varphi$, una FD que concuerde con esta hereda las propiedades A. La restricción del movimiento A después de $\mathrm{A}^{\prime}$ se puede mantener con la salvedad de que una dependencia A que involucre $\mathrm{X}$ no puede seguir una dependencia de $\mathrm{X}$ que es solamente una dependencia $\mathrm{A}^{\prime}$ (A después de $\mathrm{A}^{\prime}$ se excluye, peor A después de la mixtura $\mathrm{A} / \mathrm{A}^{\prime}$ es posible).

\subsection{Rasgos incompletos/no especificados}

Por otro lado, Martins y Nunes (2010) explican el hiperascenso en el portugués brasilero de casos como el siguiente:

$\begin{array}{cllll}\text { (27) Os meninos } & \text { parecem } & \text { que } & \text { viajaram } & \text { ontem } \\ \text { 'Los niños' } & \text { parecer-3PL } & \text { que } & \text { viajar-3PL } & \text { ayer }\end{array}$


Los autores siguen la propuesta de Ferreira $(2000,2004,2009)$ en que T de la cláusula incrustada no puede asignar caso a la FD por encontrarse incompleta en rasgos $\varphi$, por lo que esta tiene que ascender al siguiente dominio, al siguiente Esp de T donde sí se le puede asignar caso nominativo. Por su parte, Nunes (2007) añade a esta propuesta que el T con rasgos $\varphi$ completos o incompletos corresponden a la especificación en $\mathrm{T}$ de rasgos de persona o número: en caso $\mathrm{T}$ con solo rasgos de número es seleccionado, la correspondiente especificación de persona será añadida en el componente morfológico por reglas de redundancia; es decir, si T solo tiene rasgo de número y es valuado como singular en el componente sintáctico, será asociado con 1era persona en el componente morfológico; si el rasgo número recibe cualquier otro valor en el componente sintáctico, luego será asociado con el valor por defecto para persona (3era) en el componente morfológico (Martins y Nunes, 2010, 147).

Así de la oración

(28) O João parece que comprou um carro novo. 'João parece que compró un carro nuevo.'

se derivaría siguiendo la anterior explicación:

(29)[TP [o João][Caso:NOM] T[N:defecto; P:defecto] parece que [TP $t$ T[N:defecto] ...]]

$\mathrm{T}$ incrustado solo tiene rasgo de número y, como tal, es incapaz de valuar el rasgo de caso de João. Por tanto, el sujeto incrustado sigue activo para la concordancia y movimiento A, y puede ser licenciado con caso por la T matriz, el cual tiene tanto rasgos de número y persona. Luego, ambos $\mathrm{T}$ muestran morfología de 3era persona singular.

Sin embargo, en términos de Chomsky, si FC es una fase fuerte y el sujeto incrustado no está en el límite de F, lo que argumentan los autores -siguiendo a Ferreira (2000)- es que $C$ selecciona una $T$ con rasgos $\varphi$ incompletos, lo cual no es una fase fuerte. Por ello, se establece una relación sonda-meta entre T matriz y el sujeto incrustado, y el movimiento de Esp de FT al Esp de Fv matriz puede ser licenciado.

\subsection{Asignación de caso a destiempo}

Fernández-Salgueiro (2005, 2008), analiza lo que él denomina Further raising, que se diferencia del Hyper raising en que la FD que asciende no concuerda en rasgos $\varphi$ con la FT matriz ${ }^{7}$. El autor propone que mientras la FT incrustada valúa sus rasgos ininterpretables $\varphi$ a través de la relación de c-comando que establece con la FD, la valuación del rasgo de caso de la FD puede ser simultánea para cierto tipo de lenguas y a destiempo para otras, lo que produce un ulterior ascenso de FD en búsqueda de caso y satisfacer el rasgo de Principio de Proyección Extendida (PPE) de FT matriz. Esto produciría que las copias que no reciben caso no sean interpretadas en FF y no sean pronunciadas (caso de pro en lenguas de sujeto nulo). En síntesis, el tiempo de valuación de rasgos de un constituyente a través de los rasgos del otro es parametrizado.

Concuerdo con la revisión y crítica de Fernández-Sánchez (2015) al análisis de Fernández-Salgueiro $(2005,2008)$ al señalar que lo que sucede en este tipo de construcciones es un movimiento $\mathrm{A}^{\prime}$ y no A; es decir, lo que
7. Las construcciones que estudia Fernádez-Salgueiro son del tipo siguiente:

Los niños parece/* parecen que han hecho los deberes.

Donde el verbo matriz en singular no concuerda con la FD plural que asciende. Fernández-Salgueiro propone que el verbo matriz establece una concordancia por defecto con la FC complemento. 
en apariencia es un Hyper raising o en términos de Fernández-Salgueiro, Further raising, la FD viene a posicionarse en una posición Á por movimiento de Foco o Topicalización, y no a una posición A.

Sin embargo, para el análisis, es importante notar un punto crucial observado por Fernández-Sánchez sobre la propuesta de Fernández-Salgueiro. Uno de ellos es el movimiento impropio ${ }^{8}$. El autor cita la oración

(30) Ángela parece que ha considerado tu propuesta.

La FD de la oración incrustada se mueve de su posición temática en el especificador de FV al especificador de FT para ser valuado en su rasgo de caso. Si se asume que la valuación de caso puede ser pospuesta o retrasada y la FD continúa moviéndose hasta que su rasgo de caso sea chequeado, este debe escapar de la fase FC moviéndose a su límite para que sea visible para una ulterior computación. Una vez que el verbo matriz se ensambla (y se mueve a T), el especificador de la FT matriz constituye el siguiente lugar donde la FD puede chequear rasgo de caso. Sin embargo, como afirma Fernández-Sánchez, hasta este punto el movimiento impropio ya se dio, pues la FD se mueve de una posición A' (Especificador de C) al especificador de $\mathrm{T}$, posición A donde se asigna caso.

\subsection{La propuesta para el Hyper raising}

Para licenciar el ascenso de FD a través de FC, apelaré al razonamiento de Martins y Nunes (2010), de Fernández-Salgueiro (2005, 2008), y de Pesetsky y Torrego $(2001,2004,2007)$ sobre la no especificación de T y su relación de valuación ${ }^{9}$ de rasgos ininterpretables con FD -y de este con FT; y del comportamiento de la FC, que no es inherentemente A' (Fong 2019; Wurmbrand 2018).

\subsection{1. uT en FD}

Pesetsky y Torrego (2001), a través de la observación de la aparición y no aparición de that en oraciones con cláusulas relativas y su relación con la aparición de auxiliar de Tiempo en ascenso de Wh- objeto pero no de sujeto, proponen que el rasgo T está presente en la FD, que es en realidad el rasgo de Caso que se valuaría:

$$
\begin{aligned}
& \text { Naturaleza de Caso (Pesetsky y Torrego, 2001, 361) } \\
& \text { Caso es }[u T] \text { en D (caso es rasgo no interpretable en D) }
\end{aligned}
$$

Esta hipótesis nos ayudaría a explicar que no estando valuado iT en la oración subordinada, FD necesariamente debe ascender para buscar una fuente de valuación.

\subsection{2. $v a l \mathrm{~T}$ en $v$}

Además, es importante mencionar que asumiré la propuesta de Pesestsky y Torrego (2007) de que los rasgos interpretables de tiempo se encuentran en el núcleo T, el cual será valuado por el rasgo ininterpretable de tiempo ubicado en el verbo finito:
8. No persigo la generalización que menciona Fernández-Salgueiro en cuanto al sujeto nulo como efecto -y como estrategia de reparación- del ascenso ilimitado de la FD y la crítica que hace Fernández-Sánchez; sin embargo, sería interesante contrastar este fenómeno de Hyper raising en otras lenguas. Solo me ocuparé de licenciar el ascenso de FD a la posición de la FT matriz.

. La traducción para valuation o to value en español es inexacta, pues 'valuar' y 'valuación' refieren al sinónimo “valorar, señalar precio" (DRAE 2019). En este artículo, cada vez que haga mención de dichos términos, me referiré a la propuesta teórica de asignar valores de rasgos morfosintácticos a los componentes que lo requieran. 


$$
\begin{array}{llll}
\ldots \text { Tiempo ... } & {[v \text { walked }] \ldots \Rightarrow} & \ldots \text { Tiempo } & \ldots \text { [walked }] \\
\text { iT }[] & \text { uT +past } & \text { iT[2] } & \text { uT +past[2] }
\end{array}
$$

Los autores explican que

en una oración finita, el rasgo interpretable pero no valuado (interpretable) iT [ ] del núcleo Tiempo busca y encuentra su meta, el rasgo no valuado iT [ ] del sujeto FD. La concordancia se produce, estableciendo un vínculo entre las propiedades $T$ de estos dos elementos (los cuales son ahora instancias del mismo rasgo). Luego de que Concordancia entre estos dos rasgos se produce, el rasgo resultante compartido sigue estando no valuado. Consecuentemente, $\mathrm{T}$ de Tiempo busca nuevamente y entra en una relación de Concordancia con el (ininterpretable) uT valuado del verbo finito. Dado que el rasgo T del sujeto FD estableció concordancia con su contraparte en Tiempo, la concordancia entre T de Tiempo y T del verbo finito resulta en valuación de T del sujeto FD (ya que son instancias del mismo rasgo). Consecuentemente, el caso estructural (i.e. uT) de FD es valuado (Pesestsky y Torrego 2007, 278)

Así, la propuesta se basa sobre la dependencia de especificación de rasgos interpretables de Tiempo en v matriz sobre la T incrustada. A continuación, resumiré los pasos del ascenso de FD y la concordancia de Tiempo para ambas cláusulas:

\section{Ascenso de FD}

1. En la oración subordinada, T busca meta v para valuar su rasgo iT, pero v tiene uT no especificado, entonces no valúa rasgo interpretable iT en T y este no puede valuar uT en FD-caso Nominativo (se establece un sistema o cadena de concordancia T-vno valuado) 2. FD asciende a Especificador de C ( $\mathrm{C}$ tiene rasgos ininterpretables $\varphi$ que necesita valuar); 3. FD asciende a Especificador de T matriz

\section{Concordancia de tiempo}

1. ESTAR valúa el rasgo iT de T y este, a su vez, valúa uT de FD (caso Nominativo)

2. ESTAR valúa uT de $C$ (asumimos que hay un rasgo ininterpretable de $T$ en $C$ ), y luego en iT de T;

3. $T$, al pertenecer a la cadena $T-v$ de la oración subordinada, especifica los rasgos $T$ en $v$, por lo que la estructura entera ya puede ser transferida a Spell-Out.

Los pasos mencionados son representados de la siguiente derivación de la oración 'María está que canta':

I. Rasgo uT no especificado en v y ascenso de FD a Esp,T

a. $\mathrm{FC}[\mathrm{C}[2 \mathrm{~T}][\mathrm{FT}[\mathrm{Maríai}[\mathrm{uT}:$; ie] $\mathrm{T}[\mathrm{iT}:$,

II. Ascenso de FD a Esp, C (valúa rasgos $\varphi$ en C)

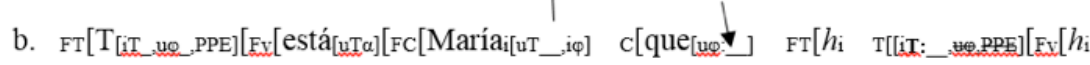
cant[uT:_]]]]]]]] 
III. Valuación de rasgo uT de FD (caso Nom), valuación de $T_{1}$, (iT de 'que), $T_{2}$ y v incrustada

c. $\quad \mathrm{FT}_{[\mathrm{Maria}}$

En (a) la FD se mueve de su posición donde se le asigna rol temático a Especificador de T para satisfacer la propiedad del rasgo de T PPE y que este valúe su rasgo de ininterpretable de Caso. No obstante, la información con los rasgos de tiempo no está especificada en $\mathrm{T}$, por lo que no se le puede asignar caso a FD ni la estructura puede ser enviada a Spell-Out, pues colapsaría. Es así que, en (b), la FD se mueve a una posición argumental, y para ello asumo que el Especificador de FC es mixta, estableciendo una ventana de escape para la FD. Es importante mencionar que el ensamble de ESTAR a la oración que describo generará en la FF la pronunciación del núcleo $\mathrm{C}$, es decir, que. Este núcleo es el que provee de rasgos interpretables de Tiempo no valuados a $\mathrm{T}, \mathrm{y}$ que $\mathrm{T}$ valúa a través de c-comando con v. En este caso, la Fv ESTAR valúa los rasgos de $\mathrm{T}$ matriz $\mathrm{y}$, de $\mathrm{C}$ de la oración incrustada (o solo los transmite a través de uT), la que transmite sucesivamente a T inferior. Finalmente, en (c), la FD asciende a Especificador de T para valuar el rasgo de Caso Nominativo y satisfacer la propiedad de rasgo PPE.

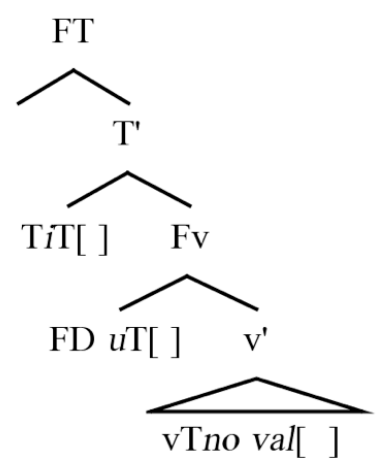

Fig. 3. Ensamble de T con Fv (uT no especificado)

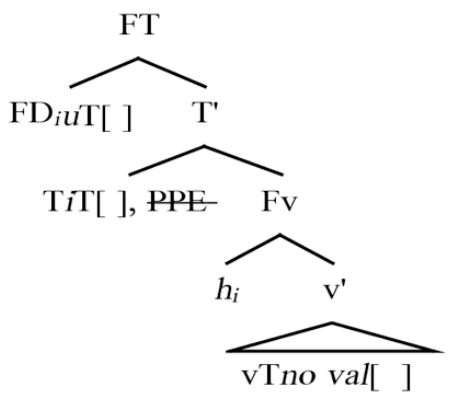

Fig. 4. Ascenso de FD a Esp, T por PPE (no se asigna caso Nom) 


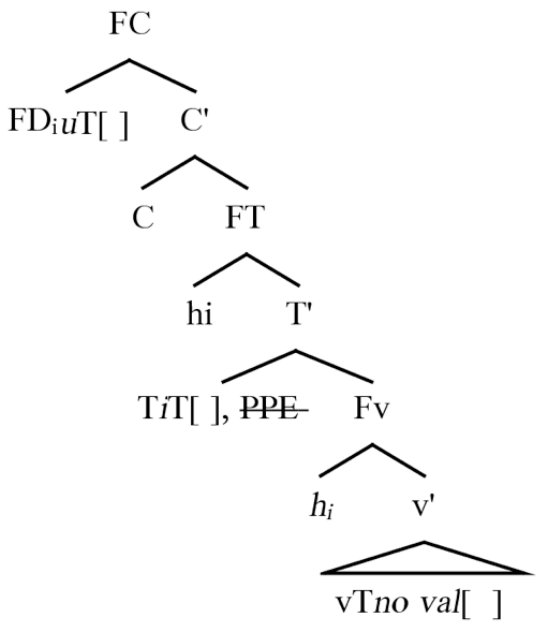

Fig. 5. Ensamble de C con FT (ascenso de FD a Esp, C argumental)

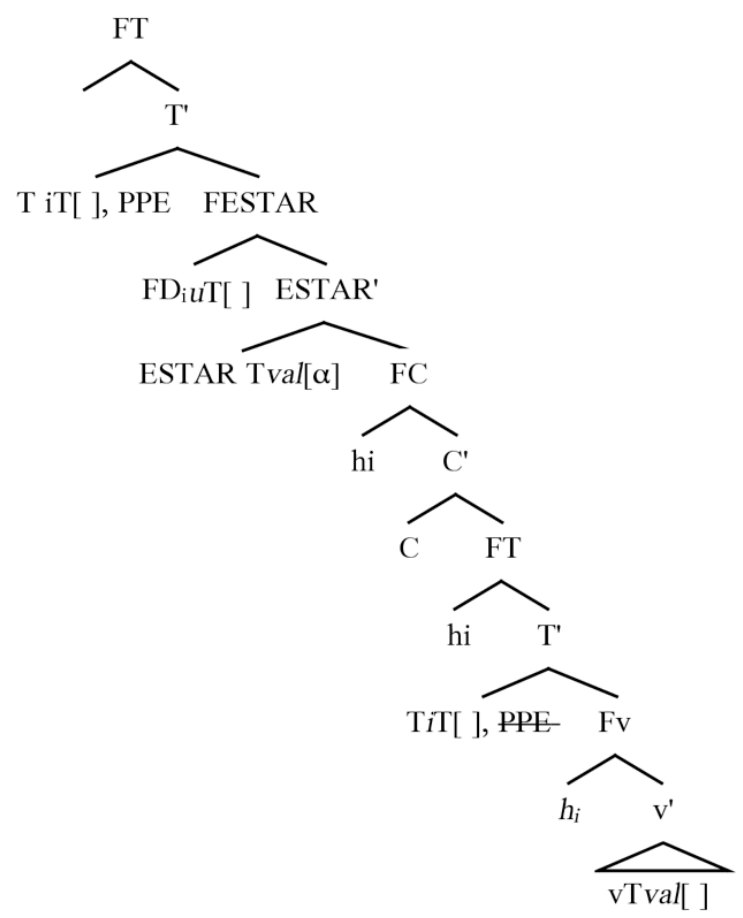

Fig. 6. Ensamble de ESTAR con FC, ensamble de ESTAR con T (ascenso de FD a Esp, ESTAR y Esp, T sucesivamente) 


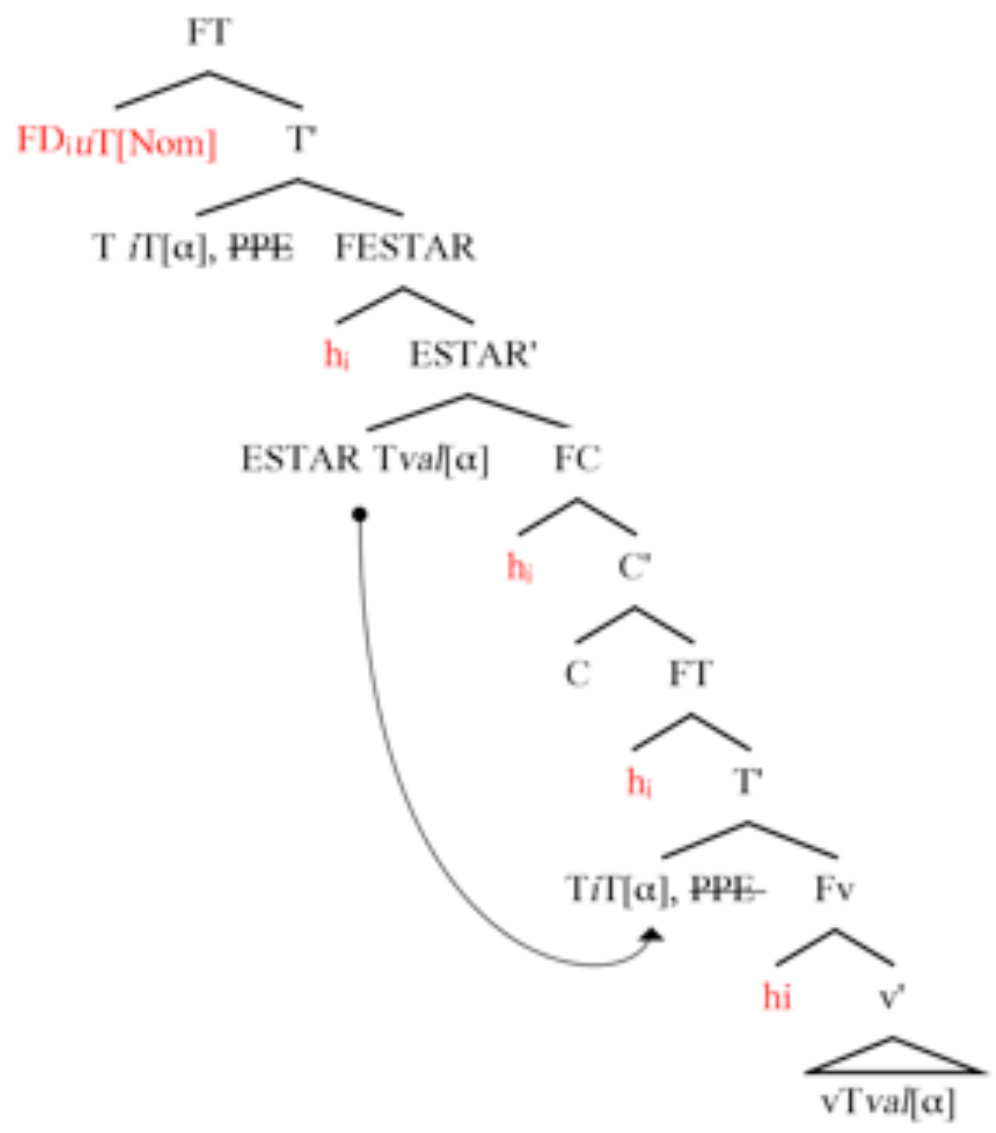

Fig. 8. Valuación de uT en FD (caso Nom), valuación de iT [ ] de T superior con ESTAR uT [a], valuación iT[ ] de T inferior con ESTAR uT $[\alpha]$

Siguiendo a Pesestsky y Torrego 2007, el rasgo uT de v inferior sería valuado al haber entrado en una relación de concordancia con $\mathrm{T}$, y esta, al ser valuado por ESTAR, sería valuado de manera simultánea.

No puedo dar una prueba concluyente sobre la condición de Esp de C como posición A o mixta A/A'. Habría que considerar otros casos en lo que este núcleo permita el ascenso u otros fenómenos que normalmente no permiten el traspaso de este dominio considerado de fase en español. No obstante, a diferencia de verbos propositivos vistos, ESTAR no selecciona una complementación proposicional, sino un evento dinámico (v). Por ello, FC entraría en la numeración con rasgos $\varphi$ que serían valuados por FD, el cual asciende sucesivamente en a la estructura.

\section{Conclusión}

En este artículo, he explicado la conformación de la estructura oracional Ella está que canta, una predicación compleja habitual en el castellano peruano. Para ello, he probado la monoclausalidad de la estructura a diferencia de cláusulas subordinadas a oraciones propositivas que involucran el complementante que. Luego, propuse que esta estructura se conforma a través de una organización eventiva por complementación: un evento estativo selecciona a otro dinámico, y ambos son interpretados desde una perspectiva aspectual de iteración. Finalmente, sustenté la explicación del 
ascenso del sujeto desde una cláusula inferior asumiendo la inespecificación de rasgo $\mathrm{T}$ del verbo inferior, con el consecuente "escape" de la FD a un nivel superior, y, para ello, asumí la posibilidad de considerar Esp,C como posición argumental o mixta A/A', dadas las propiedades de selección de ESTAR. Ante ello, queda abierta la pregunta sobre los casos en que el complementante puede poseer posiciones argumentales, ¿puede, en todo caso, el verbo "matriz" determinar los rasgos del complementante? Tal parece ser el caso de algunas construcciones del inglés y español:

Inglés

(30) a. I declare that this will be the institutional day. b. *I declare if this will be the institutonal day.

(31) a. I wonder whether or not Bill will come. b. *I wonder that Bill will come.

(32) a. I ask if there is a possibility to apply. b. "I ask that there is a possibility to apply.

(33) a. I demand for Johnny to be accepted. b. "I demand whether or not Bill will come.

Español

(34) a. Me indicó que vendría. b. *Me indicó si vendría.

(35) a. Me pregunto si vendrá. b. *Me pregunto que vendrá.

De esta manera, los verbos matrices en 30-33 y 34-35 determinan el rasgo de interrogación/declaración de sus complementos y sus morfemas $\mathrm{C}$ de núcleo; de la misma manera, podría asumir que ESTAR determina los rasgos $\varphi$ en FC de manera que la FD pueda ascender a esta posición sin problemas. Sin embargo, si la sintaxis es una maquinaria que ensambla elementos de abajo hacia arriba, es decir, en este caso, primero las cláusulas subordinadas, ¿cómo puede la cláusula superior determinar los elementos de la subordinada?

Finalmente, este estudio pone la atención en el castellano, no solamente por la forma dialectal peruana de crear, a partir de constituyentes simples, construcciones complejas para expresar formas aspectuales novedosas -y de lo cual es necesario contar con un registro-, sino también llama la atención sobre las lenguas pro-drop en general, pues abre la posibilidad de considerar el hiperascenso como rasgo dentro del parámetro de las lenguas que no requieren un sujeto materializado en el especificador de Tiempo. 


\section{Q Bibliografía}

" Amberber, Mengitsu; Brett Baker y Mark Harvey. 2010. "Introduction: Complex Predicates". En Complex Predicates. Cross-linguistic Perspectives on Event Structure, editado por Mengitsu Amberber, Brett Baker y Mark Harvey, 1-12. Cambridge: Cambridge University Press.

» Bril, Isabell. 2004. "Complex nuclei in Oceanic Languages. Contribution to an areal Typology”. En Complex Predicates in Oceanic Languages, editado por Isabell Bril \& François Ozanne-Rivierre, 1-48. Berlin: Mouton de Gruyter.

"Butt, Miriam. 2003. The Structure of Complex Predicates in Urdu. California: CSLI Publications

» Cuervo, María C. 2003. “Datives at large”. Tesis de doctorado, MIT.

»Cyrino, Sonia. 2010. "On Complex Predicates in Brazilian Portuguese”. Iberia: An International Journal of Theoretical Linguistics. vol 2.2, 2010, 1-21. Fecha de consulta, 14 de febrero de 2019. <https://idus.us.es/xmlui/handle/11441/72663>

" Fernández-Leborans, María Jesús. 1995. "Las construcciones con el verbo estar: aspectos sintácticos y semánticos”. Verba 22: 253-284.

》Fernández-Salgueiro, Gerardo. 2008. “The Case-F valuation parameter in Romance”. En The Limits of Syntactic Variation, editado por Theresa Biberauer, 295-310. John Benjamín Publishing Company.

» Fernández-Salgueiro, Gerardo. 2005. "Agree, the EPP-F and Further-Raising in Spanish". En Theoretical and Experimental Approaches to Romance Linguistics, editado por Randall S. Gess y Edward J. Rubin, 97-108. John Benjamín Publishing Company.

" Fernández-Sánchez, Javier. 2015. "An (only apparent) case of hyper raising in Spanish". Iberia: IJTL 7, 1-32. Fecha de consulta, 16 de febrero de 2019. <https://idus.us.es/xmlui/ bitstream/handle/11441/72651/268-8279-1-PB.pdf >

" Fong, Suzana. 2019. "Proper movement through Spec-CP: An argument from hyperraising in Mongolian". Glossa: a journal of general linguistics 4(1): 30. 1-42. Fecha de consulta, 20 de febrero de 2019. doi: http://doi.org/10.5334/gigl.667

" Grano, Thomas. 2015. Control and Restructuring. Oxford University Press.

"Levin, Beth y Malka Rappaport Hovav. 2004. "The Semantic Determinants of Argument Expression: A View from the English Resultative Construction". En The Syntax of Time, editado por Jacqueline Guéron y Jacqueline Lecarme, 477-494. Massachusetts: MIT Press.

"Lomashvili, Leila. 2011. Complex Predicates. The Syntax-Morphology Interface. John Benjamin Publishing Company.

"Martins, Ana Maria y Jairo Nunes. 2010. "Apparent Hyper-raising in Brazilian Portuguese: Agreement with Topics across a Finite CP”. En The Complementizer Phase. Subjects and Operators, editado por E. Phoevos Panagiotidis, 143-165. New York: Oxford University Press.

» Nash, Léa y Pollet Samvelian. 2016. “Introduction: Approaches to Complex predicates”. En Approaches to Complex predicates, editado por Léa Nash y Pollet Samvelian, 1-19. Leiden: Brill

» Nolan, Brian y Elke Diedrichsen. 2017. "Argument Realisation in Complex Predicates 
and Complex Events". En Verb-Verb constructions at the syntax-semantic Interface, editado por Brian Nolan y Elke Diedrichsen, 1-11. John Benjamin Publishing Company.

» Nunes, Jairo. 2009. “Brazilian Portuguese under Minimalist lenses”. En Minimalist Essays on Brazilian Portuguese Syntax, editado por Jairo Nunes, 3-15. John Benjamins Publishing Company.

"Pesetsky, David y Esther Torrego. 2001. "T to C movement: causes and consequences". En Ken Hale: a Life in Language, editado por Michael Kenstowicz, 355-426. Cambridge: MIT Press.

»Pesetsky, David y Esther Torrego. 2004. "Tense, Case, and the Nature of Syntactic Categories”. En The Syntax of Time, editado por Jacqueline Guéron y Jacqueline Lecarme, 495-538. Cambridge: MIT Press.

»Pesetsky, David y Esther Torrego. 2007. “The syntax of valuation and the interpretability of features”. En Phrasal and Clausal Architecture Syntactic derivation and interpretation. In honor of Joseph E. Emonds, editado por Simin Karimi, Vida Samiian y Wendy K. Wilkins, 262-294. Amsterdam/Philadelphia: John Benjamins Publishing Company.

» RAE. 2019. “Diccionario de la lengua española”. Fecha de consulta, 13 de marzo 2019. https://dle.rae.es/?id=DglqVCc

»Wurmbrand, Susanne. 2018. “Cross-clausal A-dependencies”. Trabajo presentado en Proceedings of the 54th meeting of the Chicago Linguistic Society (CLS 54).

»Wurmbrand, Susanne. 2016. “Complex predicate formation via voice incorporation”. En Approaches to Complex predicates, editado por Léa Nash y Pollet Samvelian, 248-290. Leiden: Brill.

»Wurmbrand, Susanne. 2015. "Restructuring cross-linguistically". Trabajo presentado en Proceedings of the North Eastern Linguistics Society Annual Meeting 45. Amherst: University of Massachusetts, GLSA.

»Wurmbrand. Susanne. 2003. Infinitives. 2da ed. Berlin: Mouton de Gruyter. 